Rasim M. Alguliev ${ }^{1}$, Rasim Sh. Mahmudov ${ }^{2}$

DOI: $10.25045 /$ jpis.v07.i1.04

${ }^{1,2}$ Institute of Information Technologies of ANAS, Baku, Azerbaijan

${ }^{1}$ secretary@iit.ab.az, ${ }^{2}$ rasimmahmudov@gmail.com

\title{
THE INTERNET AS A DRIVING FORCE OF THE DEVELOPMENT OF CIVILIZATION
}

\begin{abstract}
The article analyzes new realities, global and virtual relations generated by the Internet in modern society. Qualitative changes occurred with the influence of the Internet on all spheres of human activity and the emerged opportunities are commented. Moreover, the perspective development directions of the Internet and the existing threats are specified in the article.
\end{abstract}

Key words: globalization, virtualization, Internet economy, virtual labor relations, cosmic Internet, biometric Internet, Internet of Things, Internet dependence, Internet crime.

\section{Introduction}

The Internet was established as a result of convergence of computers in the 1940s, and of communications and computer technologies in the 60s of the previous century. The idea of creating the Internet network is attributed to American Scientist J. Licklider. In his concept of "Galactic Network" written in 1962, he proposed the idea of global network creation constituted of mutually linked computers. The author has shown the possibility of gaining access to data and programs in any computer by anyone via this network. Essentially, this concept was close to modern mission of the Internet. Due to these contributions in particular, J. Licklider is fairly regarded as "the father of Internet" [1].

This convergence has provided the more rapid dissemination of information over the space and has carried out the memorization of information, and solved the time issue. By the development of ICT, these capabilities have rapidly increased. Any information freed from time and space limitations is inserted to the Internet, and everyone is able to use these sources. Consequently, Internet becomes the world information storage (memory).

Internet has facilitated the transformation from industrial society to information society phase of the humanity. The main purpose of the information society as an ideology of the millennium is to satisfy the information needs of all people, regardless the age, religion, language, race, space and time, create the environment of social communication, and publicize the thoughts. By realizing these duties encountered to the information society, the humanity will achieve an instrument possessing marvelous and phenomenal features for elimination of global socioeconomic and moral problems inherited from millenniums - poverty, deprivation and illiteracy, health protection, the provision of democracy expansion, human rights and other human problems.

One of the authors of the concept of Information society, a prominent American Sociologist E.Toffler, analyzes the trends of revolutionary changes influencing the development of civilization in his work "The Third Wave". He divides the stages of society development into 3 waves agrarian society, industrial society and information society. E.Toffler mentions the increase in importance of information and knowledge exchange in information society, the decrease in prominence of traditional production factors - labor, land, raw materials and the capital, the establishment of electronic information as a main exchange instrument instead of traditional money, and the emergence of wars for information reflecting goods and services information [2].

Although the Internet has emerged as a technological novelty, it has caused an "earthquake" within the information world formed throughout the history. The wide facilities created by this global network lead to substantial changes in the way of thinking, communication, activities and social relations of people.

According to the data of the end of 2015, 3,3 billion Internet users (the world population constitutes 7,2 billion people) exist in the world. If only $18 \%$ of world households had an access to Internet in 2005, this indicator has reached $46 \%$ in 2015 [3]. 
Internet technologies develop with high speed and pledge great prospects. This development process is not amenable for forecasting in most cases. Thus, Internet is a phenomenon that its development, prospects, influences, threats are impossible to forecast accurately. The progressive novelty introduced by the Internet to the society also entails specific problems. The humanity benefits from the positive influences of the Internet, and must be protected from its negative impacts.

\section{The capabilities of the Internet}

Internet and globalization. The process of globalization is accelerated with the impact of the Internet. This digital technology reflects gigantic information and knowledge potential and leads to unity of the humanity in a integrated global society by overstepping the national-cultural boundaries. The large opportunities facilitated by the global Internet network cause substantial shifts in the way of thinking, working manner, communication and social activity. Although the radio and the television which played a prominent role in globalization in second half of the past century have eliminated the intra-country language, cultural, folk and other. differences, Internet is about to completely eradicate these differences [4].

Internet creates new possibilities for the resolution of problems associated with asymmetric information dissemination emerged throughout the civilization history; the barriers and boundaries among the countries and nations are evoperated. The process of information and knowledge exchange among various regions, states and nations was proceeding very slowly in times of weak communication. Hence, the differences related to information asymmetry among the world countries were clearly observable. However, owing to the facilities created by ICT, including the Internet today, the exchange of information and the knowledge is being expanded among people.

Internet conforms the circumscribed spaces with social, cultural, physical and functional features with one another. The global network is rapidly expanding day by day, and it is being applied to the increasing number of fields. Internet enters the world community by transgressing a particular country, and thus, the events occurring within a country are illuminated at a global scale.

The Internet is subject to various changes with the development of the globalization,. It gains new forms of existence and develops the adaptation mechanisms against crises and social convulsions. In the first place, the Internet shapes the world information space and constitutes the technical-social basis of the globalization. At present, the globalization must be considered as a process developing due to the increasing impact of the Internet on various spheres of the society. The globalization process occurring as a result of renovation and alteration of these spheres entails the formation of new lifestyle and the rapid expansion of own boundaries.

The globalization trend deeply penetrates the socio-political sphere of the society. With the development of modern Internet-communication tools, a favorable condition is created for freedom of choice of individual lifestyle. A person gains an opportunity to spread his/her ideas and find like-minded people with no dependence on anyone and anything.

Internet and virtualization. The essence of the virtuality created by Internet influence is that people counteract not with physical objects, but their images and symbols. As one of the main factors of societal transformation and formation of information society, the virtualization is accompanied with the transformation of types of human activity and the transition of those to virtual environment.

With Internet influence, the virtual "twins" of real creatures emerge in the world; the education is superseded by electronic education, traditional science with electronic science, usual citizen with electronic citizen, traditional government with electronic government, traditional commerce with electronic commerce and etc. Therefore, the world is integrated as a result of unity of these "twins", and imperfections (problems) inherited from the past are eliminated. 
In the globalization process, the virtual reality - virtual management of events and processes, plays a prominent role. In the environment of virtual reality, the opportunities emerge for changing, combining information flow, and generating new one. The virtual reality is becoming a sociological model, capable of explaining several factors and trends of the modern society. The globalization is virtualized by the influence of the Internet per se.

With the Internet impact, the traditional forms of mutual communication alter and consequently, horizontal links emerge among the participants of virtual reality. By mentioning this trend in his "Adaptive corporation" work, E.Toffler writes that "superindustrial" society type emerges with the impact of these processes and traditional organizational types lose their effectiveness [5].

Human needs to be capable to adapt to the trend of rapid changes of social infrastructures in a society. People "migrate" to virtual reality more and more with the effect of the Internet. This affects their worldview, behaviors, and lifestyle.

The virtualization also reflects new communication forms acquired by people for the realization of their unfettered creativity opportunities. As a result, a human reflects himself as a social being. Hence, the virtual society acts as an alternative to the physical society. Human is not limited in his actions here.

In general, the main three features of virtualization caused by Internet can be distinguished [6]:

- Non-material character of impacts (images give a material effect);

- Arbitrariness of parameters (objects are artificial and variable);

- Temporariness (entry-exit independence maintains the opportunities to stop and resume the existence).

Internet and economy. The impact of the Internet on economy is substantial. As a result of this impact, economic-financial system is exposed to serious changes; crises emerge at regional and global scale, profound disturbances occur in association with economic security of countries. Information and knowledge-based economy is formed.

With the emergence of the Internet, an opinion, "Massive standardized production will be superseded by the new individualized labor system based on intellectual activity with application of informatics and super technologies" stated by E.Toffler is reinforced [2]. He noted that the expansion of individual and group creative activities and the rational exchange of the results of those via Internet will take place instead of the dominance of large corporations in the economy. Indeed, the establishment of social relations in this direction facilitates the provision of more rational, equivalent opportunities of social exchange. The simpler the exchange structure, the clearer and more transparent are the relations among the producers, and the shorter is the distance till the final user. In this case, traditional commerce intermediaries (brokers, real estate agents) are not required; this function is shifted to Internet. In turn, the bigger and more complex the social organization, the more complex is its objective analysis. This situation also creates a ground for manipulation.

In the Internet economy, personal computer becomes the main production tool, and personal web site becomes an instrument for realization of produced intellectual product. Naturally, in such economy, there will be no place for private entrepreneurship (as a field of manipulative activity) not associated with professional activity and oriented to collecting social dividends.

Internet and commerce. Internet and computer eliminate several important attributes of industrial society, including cinemas, book stores, traditional trade, communication systems. Especially, as a result of development and comprehensive use of electronic commerce, it is expected that the image of cities will alter significantly. Even a usual town store outsteps the limited framework and becomes not an instrument of satisfying the needs of residents, but a global store. Because, it is possible to access the web-site of store from any corner of the world and familiarize oneself with and purchase proposed products. Moreover, there exists no such thing as the closing time. The store is always available for a customer at any time in 24 hours in a day [1]. 
The emergence of electronic commerce helps to solve one of the important problems posed to humanity so far. This is the problem of information asymmetry in markets. As seller is not aware of a potential buyer in advance, a buyer also faces the problem of lack of information about a seller. Hence, the disruption of information balance has always constituted the pith of commercial relations. Electronic commerce leads information asymmetry towards symmetry, by which the information abundance is created between the buyer and seller.

Nowadays the electronic commerce has become an inseparable part of every state economy. If people were cautious about the purchase via Internet only few years ago, it is very hard to find a person, who hasn't experienced electronic commerce operations now. The number of electronic commerce operations is rapidly increasing and influencing the world economy to a significant extent. At present, the growth pace of electronic commerce exceeds the pace of trade growth as a whole [7].

Internet and labor relations. The emergence of computer and the Internet supersedes virtual relations with traditional relations and prioritizes the intellectual labor. With the influence of information, all market types, including labor market, are subject to transformation. As a result, fundamental changes and novelties emerge in labor division, employment and the structure of profession, the labor feature, employer-employee relations, and the expansion in informationintensive labor is observed.

In case of virtual labor relations, the people can organize not only their own tasks, but also their activities by considering private interests. If an individual is not obliged to go to work every day and is able to work anywhere, he can choose more favorable living place for himself. In some regions of Europe, the flow of countrymen to cities observed during 200 years has weakened and, even, tendency of returning to countryside takes place. Based on this fact, the start of destruction of city civilization is conceded [8].

Internet and public administration. The character and nature of public administration alters in Internet environment. The establishment of electronic government is associated with optimization of services provided to citizens and business sector, the participation of all citizens in public administration, the transparency of public administration and accountability of government bodies to citizens. The essence of new administration concept is such that the governance activity is softer, better structured and of limited normalization and it is based on not vertical, but horizontal links.

Internet and scientific activity. Internet has brought innovations and dynamism to fundamental scientific research fields. As a result, traditional science is superseded by electronic science.

Electronic science is a concept carrying out the joint operation of scientific entities and organizations engaged in solution of scientific problems and possessing necessary informationcommunication infrastructure, with access to scientific-technical information and computation resources via high-speed Internet network [9].

During scientific activity, it is required to collect, memorize, process (i.e., to compute, draw logical conclusions, propose opinions, analyze and synthesize), to introduce it in required form vast amount of information, in other words, to publish it, visualize, to transmit and so forth. Internet has created previously nonexistent unique opportunities in order to carry out all of these. Owing to Internet [1]:

- scientific activity is globalized, acquired information is distributed;

- e-publications, web-sites supersede the traditional scientific publications;

- the network of electronic scientific libraries is established;

- special online environments are created on different scientific fields.

Consequently, e-history, e-geography, e-medicine, e-astronomy and etc. emerge. While memorization and processing capabilities of people are limited, the traditional historical research on space and time parameters are very complex and require substantial efforts. However, all 
information is inserted to computer memory in e-history, the historical discrepancies, inaccurate information is detected with specific processed research algorithm by computer without space and time limitations, historic facts are improved, and the truth is revealed.so forth

Internet and education. Internet education alter the traditional role of teacher. A teacher does no longer act as a sole source of information and knowledge. A part of this function is shifted to the Internet and monopoly of a teacher in this sphere is eliminated. The teacher adopts a role of technician, consultant and coordinator. For the effective use of Internet in education, the teachers are required to possess skills such as working with electronic mail, online-communication, information search systems, placing information on the Internet, the web-design and using webdesign technologies [10].

Internet technologies make education accessible for everyone. Therefore, individuals with physical and social disabilities were not able to benefit from traditional education systems. The concept of distant education based on Internet technology facilities eliminates this problem. For example, individuals with health disabilities, living far from education institutions or employed, isolated from public places or not allowed by their confessions are able to use the facilities of distant education.

Internet and medicine. Internet also penetrates the healthcare system and medical field. Wide application of Internet facilities in informatization of healthcare system and solution of medical problems acts as one of important trends in building of information society. Also, the establishment of information system regarding citizens' health, the detection of disease geography with help of GIS-technologies, monitoring, diagnostics of diseases, creation of database on pharmacies and medicaments, the provision of online medical services to patients, statistical information regarding quantitative and qualitative indicators of doctors' performance, the creation of web-portals reflecting the information regarding the treatment methods and tools of diseases and other issues are the integral components newly formed electronic healthcare [4].

Intensive application of the Internet in healthcare rapidly alters the diagnostics and treatment methods, the forms of mutual communication of a doctor and a patient, and the organization of health recovery processes. Internet becomes a prominent tool providing the accessibility of medical services for the patient. At present, the application of capabilities of Internet technologies is growing day by day for the conservation and protection of human health, the increase in birth rates and the reduction in mortality.

Internet and cultural sphere. Internet also influences the cultural sphere. Global network facilitates the access opportunities to information and knowledge resources in this sphere and causes the emergence of new cultural traditions. Internet eliminates the obstacles present at geographical distances and strongly supports the transparentizing of cultural policy by stimulating the emergence of new forms of demand in cultural sphere.

Virtual museums are one of the appearances of this tendency [11]. As a result of facilitation of access opportunities to art works and exhibitions, based on the use of interactivity and hyper environment and the application of educative mechanisms, virtual museums contribute to comprehension of cultural values and formation of new forms of comprehension.

Libraries enable online access to bibliographic data bases via Internet, and they also gradually transform their collections into electronic versions. Rare and valuable works kept in physical form in library funds have always faced the threat of destruction. By transforming them into electronic form, this risk is minimized.

Several major libraries of the world pursue the programs on transformation of old, hard copy collections into digital form. The archives of digitally published works are also established.

In general, the Internet enables the development of culture in two directions [1]:

Firstly, national boundaries and language obstacles of the culture are eliminated; the boundaries between the cultural forms such as science, education, art and recreation are dismantled. 
Secondly, each individual is not confined with comprehension of the cultural content only, but also gains an opportunity to influence it.

Internet and political activity. The role of Internet-technologies in current political process is rapidly expanding. Modern information technologies, especially the Internet, created new opportunities for active performance of parties and political leaders. The growth in use of Internet facilitates the more active participation of citizens and groups in development of political decisions and decision-making process. Moreover, Internet technologies are used by political parties as a political communication instrument. The political activism outsteps the space of traditional activities and shifts to virtual environment.

The society experiences the period of transition from representative democracy to direct, digital democracy, also from strict democracy to flexible democracy (for instance, wiki-democracy). Experts reckon that the traditional democracy has reached a critical point, i.e. there is no potential for developing democratic processes with traditional methods. Hence, the idea of "electronic democracy" is proposed as a solution. It is noted that in current state of globalization and political transformations, it is impossible to disregard the impact of Internet on political processes. Two points are indicated regarding the impact of the Internet on development of democracy: i) information accessibility; ii) the decision-making ability by analyzing those. Naturally, Internet creates unique, previously impossible opportunities for information collection [4].

Internet shapes the behavior of an active part of civil society by orienting towards the public opinion, and influences the social consciousness. Internet shapes the information environment of activities of leaders and parties, and becomes an integral part of political communications. The global network acts as a real interactive channel for communication with population and an instrument of political influence on electorate by political parties.

The social networks, historically existing at different levels and expressed with various names, has reached the new quality level by shifting to Internet environment. Separate social groups are becoming a prominent factor in the society by obtaining the opportunities such as operativeness, flexibility, mobility and informativeness in virtual space. Virtual social networks, created by people gathered around various interests, act as an important component of sociopolitical, socio-economic and cultural life of the society.

Internet creates new network societies by encompassing the earth. Prominent Spanish sociologist, the researcher of information society M. Castells assumes that the networks linking people, organizations and governments with one another will play an important role in the information age. In his opinion, economy, the labor, employment, culture, politics, state institutions, and time and space factors will consequently experience the transformation in information society [12].

\section{Internet Prospects}

Biometric Internet. The application of biometric technologies in computer networks and Internet, due to simplicity, economy and reliability, has several advantages. Firstly, biometric technologies promise wide perspectives in terms of more trustworthy personal identification. Cloud technologies have an irreplaceable role in strengthening of the global Internet network security, effective struggle against cybercrime at national and international levels, reliable performance of electronic commerce. With the help of biometric identification system, the more efficient fight against hackers, spammers and other cybercrime acts will be facilitated [1].

Sound biometry plays a prominent role in terms of maintenance of information security on the Internet. Thus, the techniques of standard information security contemplate the presence of physical carriers (passport, plastic card and etc.) or the information evidence (password, code). These approve the right to use particular information resources by an individual. A human voice acts as a unique guarantee of personal identification in sound biometry system. Unlike a password, 
code, passport, plastic card and other traditional methods of personal identification, it is impossible to lose voice features, transmit it to another person, forget, steal or falsify.

Cosmic Internet. In the near future, interplanetary apparatus will facilitate the transmission and reception of information in real time regime with the speed enabling to view video. Contemporary ships and outlet apparatus transmit information to Earth via phone line at the speed comparable to Internet connection speed. Hence, the reception of videos sent from apparatus in Mars takes several hours.

As expected, this new network technology will substantially ease the information exchange among the participants of cosmic experiment. Researchers hope that the creation of cosmic Internet will facilitate the realization of a new cosmic mission.

In this sense, the information in modern satellite networks are translated via satelliteretranslators and aboveground stations, which cause delays. IP-routers assembled on the Orbit allow the realization of direct traffic exchange among satellites. This allows to save time spent on translation and prevents the risks of losing separate packages. Moreover, it is possible to access Internet at places without cables and $3 \mathrm{G}$ networks with the help of satellite-routers [1].

Cloud technologies. Internet provides new services as it develops. Internet creates a communication environment among people and meets information needs of them. At recent times, Internet has started to satisfy people's need for computation. Thanks to the technology called "cloud computations", the access opportunity to software, data bases and other services is facilitated with various devices. Cloud technologies eliminate several technical and economic obstacles, and are able to expand the operation capacity of information technologies. The elimination of those obstacles will facilitate the contributions to information technologies market by the experts and the users to benefit from those opportunities.

According to expert forecasts, the cloud technologies will exhibit the substantial impact in society and economy as one of the most decisive technologies in the next 20 years. The rapid enhancement of possibilities of data processing, storage and transmission establishes the basis of cloud technologies economy.

The development and wide application of cloud technologies promise several economic perspectives at micro and macro levels. For this reason, the cloud technologies are considered as one of fundamental and perspective segments of information economy and Internet economy as a leading trend of it. Hence, several leading world countries devote a special place to cloud technologies in national strategies and programs on development of information society, ICT, information economy or the Internet [13].

Internet of Things. At the next development stage of the Internet, the boundless expansion of opportunities of these global networks and fundamental changes in the their essence are expected. Today's Internet is constituted of the global network of computers used by people. At the next development stage of the Internet, the connection of all useful things (household devices, electric devices, daily consumer products, transport vehicles, production equipment, labor tools, information carriers, medical tools, security and control systems, fauna and flora) to global network, and the establishment of Internet of Things is expected.

Internet of Things is characterized as the next revolutionary development stage of the industry of information technologies after computer, Internet and mobile phone communications. If this concept is brought into life, all useful things surrounding us will have IP address in the near future. According to forecasts, the Internet of Things will penetrate the lifestyle of people ubiquitously and in all aspects, and alter their life significantly. The number of users of the Internet of Things will reach 2 billion people and the annual income, earned from the network, will be 800 billion dollars in 2020. According to another forecast, the number of terminals connected to Internet will reach 50 billion worldwide. Also according to expert estimations, the market volume of RFID constituting the technological basis of Internet of Things will amount to 27 billion dollars 
in 2018. For comparison, it can be mentioned that this number was equal to 1,5 billion dollars in 2004 [14].

The solution of several important problems is expected with realization of network of the Internet of Things. Furthermore, the quality improvement of medical services, the more reliable maintenance of public security, the issues of upgrading administration processes will reach the solution. As a whole, the successful realization of Internet of things technologies is expected to enable the improvement of standard of living of people, the creation of new and more favorable job places, the creation of new opportunities for business and the increase in productivity and competitiveness in production.

Big Data technologies. Big data is a general term used for the illustration of large-volume unstructured and semi-structured data generated by a particular state, state institutions and private organization. The processes of large-volume different-format data processing, analysis and extraction of useful knowledge from those are carried out by Big Data technologies.

The Internet network is a major data base, that is, the largest object of Big data and raw material base. The information of larger scale is currently generated in Internet services, social networks, and e-commerce systems. According to calculations, the amount of information sent by e-mail is equal to 2,9 million per second in the world. The volume of downloaded videos from YouTube per minute constitutes 20 hours. The information of 24 petabyte volume is processed by Google during the day. 50 million information is posted on Twitter during the day. The minutes spent on Facebook per month reach 700 billion. The number of products placed on Amazon per second is equal to 73 items. 300 million photos are uploaded to Facebook in one day [3].

All these facts show that Internet acts as a primary information source for development of Big Data technologies. Several Internet enterprises process and use large information massives gathered regarding their performance via Big Data technologies, and introduce those to other administration and business-oriented organizations on commercial basis. Moreover, the number of Internet companies, specifically engaged in marketing activities, increases day by day.

\section{The threats of Internet}

Information Security. The provision of security of Global Internet Network is an important issue for its sustainable and reliable performance. The information security becomes the main factor in Internet environment. Internet is a suitable arena for intelligence, sabotage, terror and protest activities. Global network is characterized by multi-branched architecture, in which largevolume information of scientific-technical, military, socio-economic and political content circulate. As a result of threats to Information Security, a serious harm can be incurred to country performance in political, economic, military and other fields, and in this case, separate citizens and the society as a whole may face substantial socio-economic harm [1].

According to calculations of American experts, the disruption of operation of computer networks used in public administration and bank structures is as harmful as the consequences of nuclear weapon used against the country.

In countries where Internet is widely used, the crime acts associated with computer systems and Internet occupies a prominent place among general law infringement acts. The rapid increase of crime acts in Internet environment is primarily related to the features of the global network openness, accessibility for everyone, the absence of control by any country or organization. Moreover, the absence of effective law regulation mechanisms related to Internet activity, the existence of jurisdiction problems, also non-professionalism of law enforcement agencies facilitates the increase in number of criminal acts in cyberspace [15].

Energy security. The majority of modern human activities are carried out with the help of computers and Internet. It is no coincidence that the failures often occurring in computer systems disrupt the work of financial, transport, production and other fields, and incur negative impact on everyday life of people. 
One of the factors necessary for normal and continuous operation of Internet is the energy security. It is to be considered that although the Internet creates a virtual world, it consumes real energy. Nowadays, 30-50 percent of costs of any computation center are constituted by energy costs. One large computation center consumes as much energy as a small town with 20 thousand people (for instance, Quba, Shamakhi) [1].

Hence, as a result of power supply shutdown, the operation of state and private corporate computer networks, automatized production and service enterprises can be paralyzed and large financial losses can be incurred.

At present, experts articulate regarding the minimization of energy consumption of the Internet. They concede that sustainable power supply is of specific topicality. For this reason, the restoration of existing energy sources and the discovery of alternative sources is one of the most important issues encountered by the humanity.

Internet addiction. One of the problems caused by Internet is the consequences of information abundance within the society. An individual becomes addicted as a result of excessive use of information and computer.. This socio-psychological condition is called "escapism". In modern society, information sources such as television, computer, Internet and mobile phones "voraciously consume" people's time, and become the most important life priorities. The volume of the information received by a modern individual during two weeks is equal to information volume received by an individual during the lifetime in the Middle Ages. The psychological reaction of an individual to rapid and radical changes occuring in society, due to the impact of these technologies, is named the "futureshock" by E.Toffler. In his opinion, the cause of shock condition is the inability of an individual to flexibly respond to new realities brought by the technology and adapt to a new situation [16].

Internet addiction is a source of danger for not only an individual, but for the society as a whole. In order to avoid the addiction to information sources and the Internet, possession of information culture and behavioral norms with information by individual is essential.

The problems associated with Internet regulation. The difficulties associated with the specification of unified regulation policy, principles and mechanisms are mostly related to these factors. Thus, each state and nation aspire to be represented by own national-moral values in virtual space. However, it is a complex issue to find the points in the Internet, where the interests of all parties are maintained. Thus, traditional national and international legal systems find it hard to regulate the realities brought by the Internet. The incentives are proposed for the realization of measures on Internet regulation at international level. Internet weakens the international legal rules and national legislation which is based on geographical boundaries. It becomes a complex issue and sometimes untenable to govern the virtual relations formed on capabilities of Internet technologies with real world rules of law. The regulation of virtual relations necessitates the development and application of new laws and norms pertaining to this environment [17].

Internet crime. Unique technological characteristics of Internet facilitates a favorable environment for committing several criminal acts. The existence of legal loopholes in the field of global network regulation perplexes the prevention of such negative cases. The Internet acts as a favorable environment for realization of both traditional criminal acts in more convenient way, and previously unknown, new types of criminal acts pertaining to the global network [1].

Internet capabilities are actively used by criminal elements; websites are widely spread, which carry out the advertising and sale of drugs and weapon, post pornographic materials and have racial, nationalistic and extremist content. The high social insecurity of crimes in such virtual space encompasses large human groups and different countries.

The rapid increase of criminal cases in Internet environment is firstly related to the features of this global network - openness, accessibility by everyone, and the absence of control by any government or organization. 


\section{Conclusion}

It is to be noted that each progressive novelty has entailed specific problems during the development history of civilization. However, the humanity has always benefited from progressive innovations and been able to overcome the problems. Internet is a phenomenon with no analogue in history and not comparable with any other instrument. Therefore, none of technological novelties were able to alter and develop the humanity as Internet is doing. Development tendencies of global networks are so dynamic and multi-branched that it is impossible to accurately forecast its prospects. As the benefits of Internet to humanity are large-scale, the negative consequences also encompass all fields. Nevertheless, the past and rich experience of humanity and present efforts show that people will be capable to eliminate the negative consequences of the Internet for the sake of better opportunities and more convenient life.

\section{References}

1. Alguliev R.M., Mahmudov R.Sh. A multi-faceted view to Internet phenomenon. Expressinformation. Information society series, Baku, "Information Technologies" press, 2010, 96 p.

2. Toffler E. The third wave. M: OOO "Publishing AST", 1999, 784 p.

3. http://www.internetworldstats.com

4. Alguliev R.M., Mahmudov R.Sh. The essence of Internet and some scientific approaches on its features // Information society problems, Baku, 2012, № 1, pp. 41-51.

5. Toffler A. The Adaptive Corporation, Gower, 1985, 217 p.

6. Berre A.J., Gómez-Pérez A., Tutschku K. Future Internet - FIS 2010: Third Future Internet Symposium, Berlin, Germany, September 20-22, 2010, Proceedings, Springer, 2010, 157 p.

7. http://www.emarketer.com

8. Mahmudov R.Sh. Distant labor relations: international practice and the perspectives of application in Azerbaijan // Information society problems, Baku, 2010, № 1, pp. 56-62.

9. Alguliev R.M., Fataliyev T.Kh. Some aspects of formation of electronic science // News of Baku State University. The series of physico-mathematical sciences, 2008, № 4, pp. 77-83.

10. Alguliev R.M., Mahmudov R.Sh. Some conceptual issues of education informationalization // News of Azerbaijan State Pedagogical University. The series of pedagogic-psychological sciences, 2007, № 9, pp. 9-16.

11. The formation and conservation of cultural heritage in information society, UNESCO Publication, 2003. Trans. from English, Saint-Petersburg, 2004, 110 p.

12. Castells M. The Rise of the Network Society: The Information Age: Economy, Society, and Culture, Vol. 1, John Wiley \& Sons, 2011, 656 p.

13. Mahmudov R.Sh. The current state of the economy of cloud technologies and the analysis of development perspectives // Information society problems, Baku, 2014, № 2, pp. 14-23.

14. Alguliev R.M., Mahmudov R.Sh. Internet of Things // Information society, № 3, pp.42-48

15. Alguliev R.M., Imamverdiyev Y.N. The incidents of information security, Baku, "Information Technologies" press, 2012, 219 p.

16. Alguliev R.M., Mahmudov R.Sh. The issues of Internet addiction and the methods of fight. Express-information. Information society series, Baku, "Information Technologies" press, 2009, 62 p.

17. Alguliev R.M., Mahmudov R.Sh. The problems of Internet regulation. Express-information. Information society series, Baku, "Information Technologies" press, 2010, 115 p. 